\title{
Generation of non-Gaussian statistics and coherent structures in ideal magnetohydrodynamics
}

\author{
M. Wan, ${ }^{1}$ S. Oughton, ${ }^{2}$ S. Servidio, ${ }^{1}$ and W. H. Matthaeus ${ }^{1}$ \\ ${ }^{1}$ Department of Physics and Astronomy and Bartol Research Institute, University of Delaware, \\ Newark, Delaware 19716, USA \\ ${ }^{2}$ Department of Mathematics, University of Waikato, Hamilton 3240, New Zealand
}

(Received 1 July 2009; accepted 29 July 2009; published online 13 August 2009)

\begin{abstract}
Spectral method simulations of ideal magnetohydrodynamics are used to investigate production of coherent small scale structures, a feature of fluid models that is usually associated with inertial range signatures of nonuniform dissipation, and the associated emergence of non-Gaussian statistics. The near-identical growth of non-Gaussianity in ideal and nonideal cases suggests that generation of coherent structures and breaking of self-similarity are essentially ideal processes. This has important implications for understanding the origin of intermittency in turbulence. (C) 2009 American Institute of Physics. [DOI: 10.1063/1.3206949]
\end{abstract}

A well-known feature of turbulence is the emergence of small-scale coherent structures that are responsible for enhanced dissipation; in steady-state these structures cause departures from self-similarity and the phenomenon of intermittency. ${ }^{1}$ Classically, the notion of intermittency can be discussed in the inertial range, and equivalently, in the dissipation range. ${ }^{1-4}$ Here we show, by comparing ideal simulations with well-resolved dissipative simulations (with identical initial conditions), that non-Gaussianity and characteristic coherent structures are initiated almost identically in the two systems. Therefore we postulate that the origins of coherence and intermittency are essentially ideal, with dissipation acting only to limit growth of the smallest scale structures.

Although we envision broader implications for turbulence, e.g., for three dimensions (3D), and for hydrodynamics (HD), for several reasons, we adopt a two dimensional magnetohydrodynamics (2DMHD) model for this study. First, 2DMHD admits a direct cascade of energy and hence produces small-scale structure more robustly than does 2D HD. ${ }^{5-8}$ Second, it is possible to attain much greater spatial resolution with 2DMHD, compared to 3D HD and MHD. Furthermore, preferred coherent structures in 2DMHDsheets of electric current density-play a central role in magnetic reconnection. ${ }^{9,10}$ 2DMHD also remains a baseline description in solar, ${ }^{11,12}$ space, ${ }^{13}$ and astrophysical plasmas. ${ }^{14}$ We recall that $2 \mathrm{DMHD}$ has a special relationship to 3DMHD. A strong uniform applied magnetic field $\boldsymbol{B}_{0}$ suppresses spectral transfer parallel to $\boldsymbol{B}_{0}{ }^{15}$ which can induce a 2D-like anisotropy. Higher-order statistical properties also become anisotropic (e.g., Ref. 16); however, further examination of this anisotropy is beyond the current scope. Here we consider 2DMHD with $\boldsymbol{B}_{0}=0$.

Our computations solve the 2D incompressible MHD equations in terms of the vector potential $a$ and vorticity $\omega=(\nabla \times \boldsymbol{v}) \cdot \hat{z}$,

$$
\begin{aligned}
& \partial_{t} \omega+\boldsymbol{v} \cdot \nabla \omega=\boldsymbol{b} \cdot \nabla j+\nu \nabla^{2} \omega, \\
& \partial_{t} a+\boldsymbol{v} \cdot \nabla a=\eta \nabla^{2} a,
\end{aligned}
$$

involving magnetic field $\boldsymbol{b}=\nabla a \times \hat{z}$, current density $j=-\nabla^{2} a$, velocity $\boldsymbol{v}$, viscosity $\nu$, and resistivity $\eta$.

Equation (1) is solved numerically in a $2 \pi$-periodic box using a Fourier spectral method with 2/3-rule dealiasing. ${ }^{17}$ The time integration is a second-order Runge-Kutta method. Initial $(t=0)$ spectra of $\boldsymbol{v}$ and $\boldsymbol{b}$ are chosen proportional to $\left[1+\left(\frac{k}{k_{0}}\right)^{8 / 3}\right]^{-1}$, within a band of wave number $k=|\boldsymbol{k}|$; phases are assigned using Gaussian random numbers. The initial kinetic and magnetic energies are equal, $E_{v}=\left\langle|\boldsymbol{v}|^{2}\right\rangle / 2=0.5$ and $E_{b}=\left\langle|\boldsymbol{b}|^{2}\right\rangle / 2=0.5$. The cross helicity $H_{c}=\langle\boldsymbol{v} \cdot \boldsymbol{b}\rangle$, initially small, remains so during the runs. Angle brackets $\langle\cdots\rangle$ denote a volume average over the box.

Simulations were performed for a range of resolutions, $k_{0}$ values, and initially excited $k$-bands. Here we report on two typical $1024^{2}$ runs, one ideal and one dissipative-with identical initial data, using $k_{0}=5$ and an initially populated $k$-band of 3-8. For the dissipative run $\nu=\eta=1 / 800$. In the ideal run, $\nu=\eta=0$. The energy $E=E_{v}+E_{b}, H_{c}$, and meansquare potential $\left\langle a^{2}\right\rangle$, are well conserved (with an error $<0.01 \%$ ). Accuracy of the dissipative run is verified with higher resolution runs.

Figure 1 shows the temporal evolution of the total energy spectra, $E(k)$. The ideal and dissipative spectra remain almost identical until $t \approx 0.1$. Subsequently they diverge, with the difference most prominent at the highest $k s$ and encroaching to lower $k$ with time. Note that at $t=1$ the ideal run has a spectrum with what looks remarkably like a $5 / 3$ inertial range for $15 \lesssim k \lesssim 100$. This is a transient effect. The clear upturn at higher $k s$ is associated with the early stages of approach to statistical equilibrium of the ideal Galerkin model. $^{18-20}$

Comparison of contours of electric current density for the ideal and dissipative cases (Fig. 2) reveals that essentially identical coherent structures are produced at early times. The 


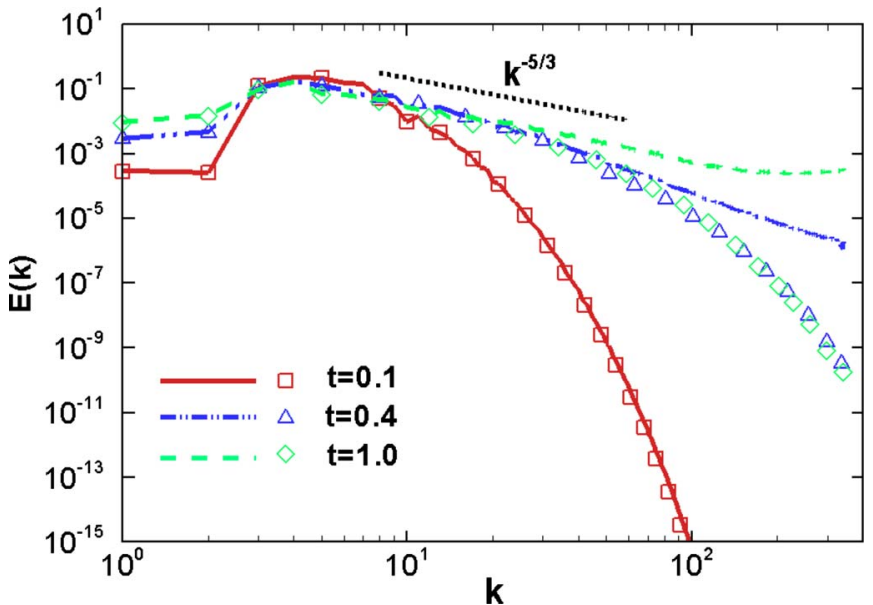

FIG. 1. (Color online) Total energy spectrum for ideal (lines) and dissipative (symbols) runs at $t=0.1,0.4$ and 1.0.

connection between ideal and dissipative dynamics was suggested decades ago ${ }^{7}$ and has been of recent interest. ${ }^{21,22}$ By $t=1$, however, the plots are radically different with small scale coherent structures much less prominent in the ideal run. The correlation coefficient between the current density in ideal and dissipative cases (not shown) remains very high $(>0.98)$ until $t \approx 0.3$, and it declines smoothly toward zero for $t>1$.

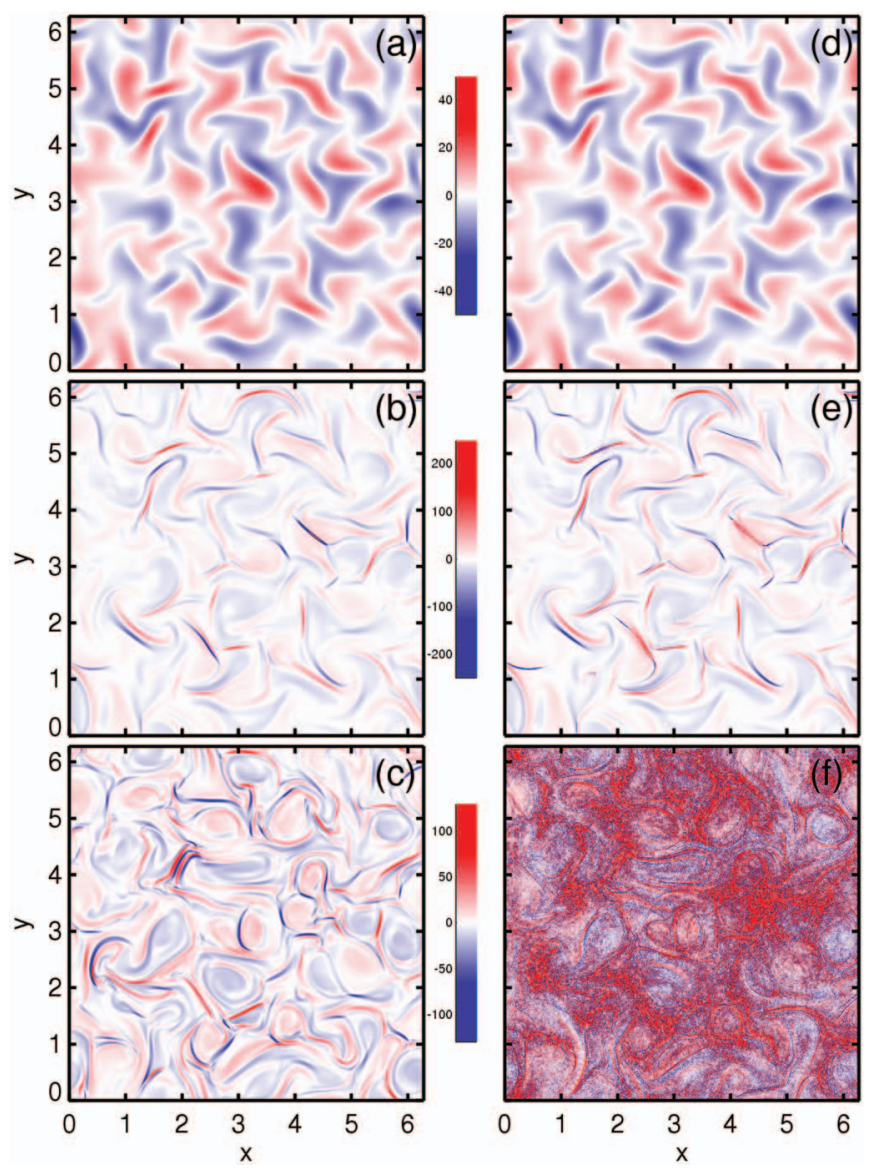

FIG. 2. (Color) Contours of the electric current density ( $j$ ) for the dissipative run (left) and the ideal run (right) at (top to bottom) $t=0.1,0.4,1.0$.
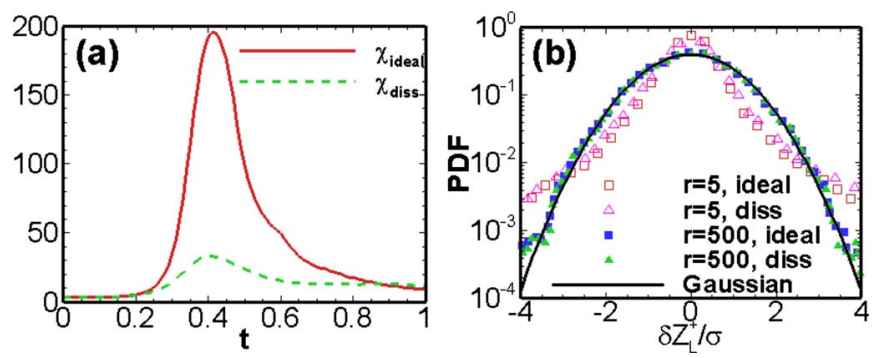

FIG. 3. (Color online) (a) Evolution of the kurtosis $\chi$ of $j$ for ideal (solid line) and dissipative (dashed line) runs. Later, $\chi_{\text {ideal }} \approx 8.8,3.7,3.2$ and $\chi_{\text {diss }} \approx 11,9.3,16$ at $t=1,2,3$, respectively. (b) PDFs of longitudinal Elsässer increments $\delta z_{L}^{+}$, for ideal and dissipative runs at $t=0.4$ for small scale $(r=5)$, and large scale $(r=500) ; \sigma^{2}$ is variance of $\delta z_{L}^{+}$.

The statistical differences between ideal and nonideal cases become apparent in examination of the fourth-order moment (kurtosis) of the current, $\chi(t)=\left\langle j^{4}\right\rangle /\left\langle j^{2}\right\rangle^{2}$. Figure 3 shows time histories of $\chi(t)$ for the ideal and dissipative runs. The maximum of $\chi_{\text {ideal }}$ is larger, near 196, or about six times its dissipative counterpart. This suggests that dissipative effects limit growth of the kurtosis. The decrease in $\chi_{\text {ideal }}$ and eventual saturation at $\approx 3$ is due to the Gaussianization of the system as excitation becomes thermalized (equipartitioned) across all Fourier modes. ${ }^{18}$ The similar decrease in $\chi_{\text {diss }}$ occurs for a different reason, namely, that the turbulence is decaying, and the Reynolds numbers decreasing. Reduction of nonlinearity ${ }^{23}$ may also play a role.

More complete information is afforded by the probability distribution function (PDF) of longitudinal increments [e.g., $\left.\delta v_{L}=\{\boldsymbol{v}(\boldsymbol{x}+\boldsymbol{r})-\boldsymbol{v}(\boldsymbol{x})\} \cdot \hat{\boldsymbol{r}}\right]$. Figure 3 shows (dissipative and ideal) increments of the Elsässer field $z^{+}=\boldsymbol{v}+\boldsymbol{b}$ for two values of separation, at time $t=0.4$. At smaller scale, both PDFs begin as nearly Gaussian distributions (not shown) and evolve toward distributions with fat tails as seen in the Figure. At larger scales the PDFs remain closer to Gaussian. At later times the ideal case returns to Gaussian at all scales. The similarity of the non-Gaussian features in the two cases at early times is a central point of this paper.

Energy transfer between scales is a fundamental idea in turbulence ${ }^{24-27}$ and involves transfer within triads of Fourier modes which satisfy $\boldsymbol{k}+\boldsymbol{p}+\boldsymbol{q}=0$. Here $\boldsymbol{k}, \boldsymbol{p}$, and $\boldsymbol{q}$ are the respective wave vectors of the three modes in the triad. To study transfer between scales, we introduce a shell filter decomposition, e.g., $\boldsymbol{v}(\boldsymbol{x})=\sum_{m} \boldsymbol{v}_{m}(\boldsymbol{x})$, where $\boldsymbol{v}_{m}(\boldsymbol{x})$ $=\sum_{|k-m|<1 / 2} \hat{\boldsymbol{v}}(\boldsymbol{k}) e^{i \boldsymbol{k} \cdot \boldsymbol{x}}$ and $\hat{\boldsymbol{v}}(\boldsymbol{k})$ is the Fourier transform of $\boldsymbol{v}(\boldsymbol{x})$. The $m$ th shell, where $m$ is an integer (in a periodic domain), is defined as all $\boldsymbol{k}$ such that $|k-m|<\frac{1}{2}$. Then the energy flux, $\Pi^{(m, n)}$, from the $m$ th to $n$th Fourier space shell, can be written as

$$
\begin{aligned}
\Pi^{(m, n)}= & \left\langle-\boldsymbol{v}_{n} \cdot(\boldsymbol{v} \cdot \nabla) \boldsymbol{v}_{m}+\boldsymbol{v}_{n} \cdot(\boldsymbol{b} \cdot \nabla) \boldsymbol{b}_{m}\right. \\
& \left.-\boldsymbol{b}_{n} \cdot(\boldsymbol{v} \cdot \nabla) \boldsymbol{b}_{m}+\boldsymbol{b}_{n} \cdot(\boldsymbol{b} \cdot \nabla) \boldsymbol{v}_{m}\right\rangle
\end{aligned}
$$

(see e.g., Ref. 25 for details). We can then define the sums

$$
\Pi^{ \pm}(k)=\frac{1}{2} \sum_{m \leq k, n>k}\left(\left|\Pi^{(m, n)}\right| \pm \Pi^{(m, n)}\right)
$$

to be the direct (+) and inverse (-) energy flux across $k$, 

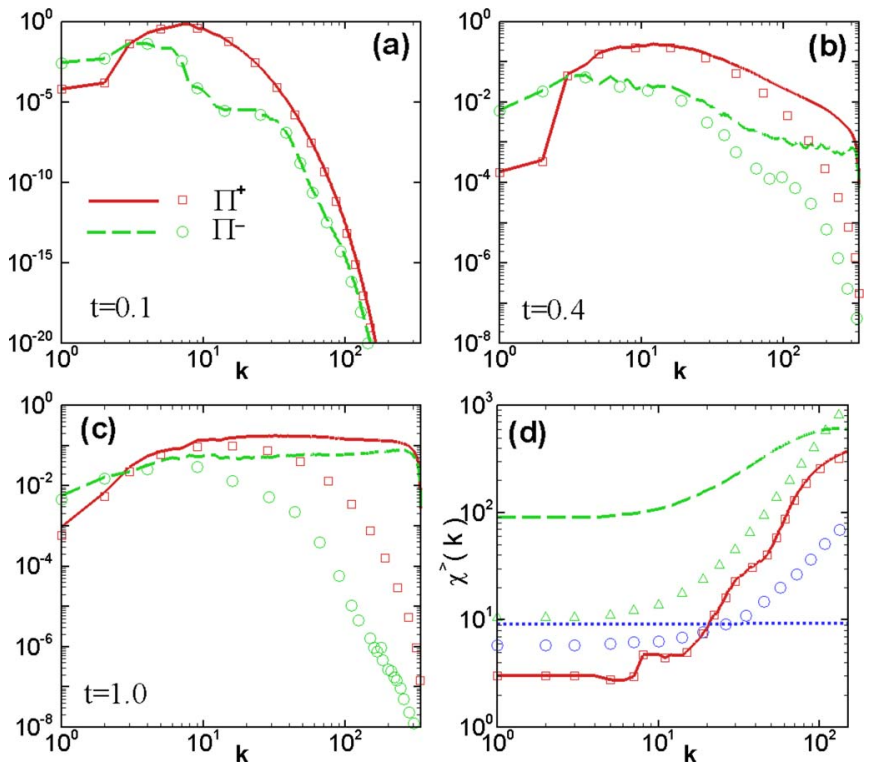

FIG. 4. (Color online) The evolution of $\Pi^{+}$and $\Pi^{-}$for the ideal run (lines) and dissipative run (symbols) at $t=0.1,0.4,1.0$, respectively. (d): scaledependent kurtosis of $j, \chi^{>}(k)$, for the ideal run (lines) and dissipative run (symbols) at $t=0.1$ (solid, squares), 0.4 (dashed, triangles) and 1.0 (dotted, circles). At $t>0.4$ the ideal and dissipative cases differ greatly-the dissipative case remains intermittent; the ideal case approaches equipartition and later become Gaussian.

respectively. These quantities are displayed at three times in Fig. 4. As panel (a) indicates, up to $t \approx 0.1$ the ideal and dissipative $\Pi^{+}$are the same, and similarly for $\Pi^{-}$. Note that at high $k$ the two fluxes are almost equal (although small). By $t=0.4$, the time of peak kurtosis in both runs, the fluxes in the ideal and dissipative cases have diverged at larger $k$. In both runs, the direct flux is dominant at almost all scales (except at $k \leqq 3$ where inverse cascade is operative ${ }^{18,19}$ ). Where the curves differ, the magnitudes are typically substantially larger in the ideal case. This remains true at $t=1$, but the ideal approach toward absolute equilibrium means that $\Pi_{\text {ideal }}^{ \pm}(k)$ have become rather flat.

From this comparison between ideal and dissipative numerical experiments, it appears that the key point for the formation of coherent structures is the energy flux through different scales. The positivity of the net flux essentially is associated with emergence of non-Gaussian statistics. In the dissipative case the imbalance between $\Pi^{+}$and $\Pi^{-}$survives because of the dissipation, while in the ideal case a backscatter of energy, due to the finite $k$-space truncation, destroys the coherency (Fig. 2). The ideal case becomes Gaussian again at later times due to this backscatter from the wave number space boundary. The early transient in the ideal case signifies that the production of intermittent structures does not require dissipation. Dissipation plays only a secondary role, damping $\Pi^{-}$and ensuring a large ratio $\Pi^{+} / \Pi^{-}$.

Another revealing quantity is the scale-dependent (or filtered) kurtosis, $\chi^{>}(k)$, defined as the kurtosis of the highpass filtered $j(\boldsymbol{x})$, i.e., Fourier components of $j$ with wave numbers $k^{\prime}<k$ are zeroed. ${ }^{1}$ (Here the time argument is suppressed.) The values of $\chi^{>}(k)$ for ideal and dissipative cases are equal for $t \leqslant 0.1$ across the entire spectrum [Fig. 4(d)]. As time progresses to $t=0.4, \chi^{>}(k)$ temporarily becomes much greater in the ideal run than in the dissipative run, except at very high wave numbers. However by $t=1, \chi^{>}(k)$ becomes flat with $\chi^{>}(k) \approx 9$ [it eventually saturates (see caption, Fig. 3) at $\chi^{>}(k)=3$ ], while the dissipative case maintains strong non-Gaussian features at the higher values of wave number. It is evident that the energy spectra (Fig. 1) remain closer to one another in ideal and dissipative cases than do the $k$-filtered kurtoses. This suggests that the phase information is modified more quickly by the backscatter from the wave number space truncation than is the value of the energy spectrum. Apparently even a small amount of backscatter can greatly modify the phase-associated statistics, rapidly destroying coherency and non-Gaussian features.

The origin of non-Gaussian statistics is due to coherent structure formation, and our results indicate that this occurs in ideal cases and (well-resolved) dissipative cases in very similar ways. Thus, the main role of dissipation is to limit the growth of the small-scale structures. This basic idea was investigated some time ago but from a different perspective by Frisch et $\mathrm{al}^{7}$ who noted that ideal MHD generates smallscale current sheets. Here we see that these are essentially the same as those produced in the dissipative case, and also that the corresponding growth of non-Gaussian statistics is quantitatively almost identical in ideal and dissipative cases, up to the time when excitation at the smallest allowed scales becomes dynamically significant.

Limitation of the growth of non-Gaussianity in the two cases is of very different origin; in the ideal case, for any practical simulation, the smallest allowed scale is imposed by the discretization cutoff. That boundary in $k$-space produces a backscattering of energy ( $\Pi^{-}$increases) that starts to thermalize all the scales. The reflection changes phase information, destroying coherence even faster than it modifies the energy spectrum. For the dissipative case, on the other hand, the limitation on non-Gaussianity is associated with the smallest dynamically significant scales, determined by the physical dissipation processes.

Non-Gaussianity appears rapidly and intensely in the initially unpopulated Fourier modes in the ideal runs, and so we suspect that the origin of this effect may be robust and insensitive to details. Consider a simplified model in which couplings to unpopulated modes (say " $A$ ") are due to quadratic nonlinearities [as in Eq. (1)]. For the nondissipative case we write $\dot{A}=B \times C$ for initially large-scale band-limited uncorrelated Gaussian random variables $B$ and $C$. At times $t$ very short compared to the correlation times of $B$ and $C$, we estimate that $A \sim B(0) C(0) t$ for initial values $B(0), C(0)$. Since $B$ and $C$ are uncorrelated, the kurtoses are related by $\chi(A)=\chi(B) \chi(C)$ and the kurtosis of the initially transferred excitation into $A$ is non-Gaussian, with $\chi(A)=9$. This simple argument captures the essential reason that early time transfer to high $k$ is non-Gaussian (see Ref. 28).

It is natural to consider the possible relationship between the present results and driven, steady, high Reynolds number intermittent turbulence, in which inertial range increments become increasingly non-Gaussian with decreasing scale. There are clear similarities-e.g., the ideal case at early 
times shows filtered kurtosis that increases monotonically with $k$, a key property that characterizes intermittency. ${ }^{1}$ Furthermore the coherent structures produced in ideal and dissipative cases remain almost identical up to a time when the smallest allowed scale is significantly excited. Based on this close similarity we suggest that the origins of coherent structure at early times are the same in dissipative and ideal cases. Therefore the interesting possibility emerges that intermittency in steady turbulence, which is a consequence of smallscale coherent structure, may be viewed as primarily driven by ideal processes and limited by dissipative processes. This idea may be relevant to discussions of intermittency and dissipation that pervade the literature (e.g., Refs. 7, 29, and 30). One might question, for example, whether the breaking of self-similarity associated with intermittency is essentially an ideal process. This would have interesting implications, yet to be explored, for understanding refined similarity, the connection between scaling laws in the inertial range and the statistics of the dissipation in both $2 \mathrm{D}$ and $3 \mathrm{D} .{ }^{31}$ In particular, in the ideal case the dissipation is undefined, yet the present results suggest that a connection persists between inertial range scalings and gradients of the coherent structures. These ideas may also be relevant to the relationship between bottleneck effects, statistical mechanics, and effective viscosity caused by eddy noise. ${ }^{21,22,32}$

Finally, the breakdown of the correspondence between ideal and dissipative cases is associated with interaction between spectral transfer and the $k$-space cutoff due to numerical discretization. Understanding this interaction is of importance in assessing the adequacy of resolution in a numerical model. We defer development of this practical application to a future publication. The essential physical content of the present study - that generation of non-Gaussian statistics is driven by ideal processes-is likely to apply beyond 2DMHD, for example in 3D HD and other systems. Preliminary 3D HD and MHD studies (necessarily at lower resolution) support this conclusion, but confirmation requires further investigation.

This research supported in part by NSF Grant Nos. ATM0539995 and ATM0752135, and by NASA Grant Nos. NNG06GD47G and NNX08AI47G (Heliophysics Theory).
${ }^{1} \mathrm{U}$. Frisch, Turbulence: The Legacy of A. N. Kolmogorov (Cambridge University Press, Cambridge, 1995).

${ }^{2}$ K. R. Sreenivasan and R. A. Antonia, J. Fluid Mech. 29, 435 (1997).

${ }^{3}$ H. Politano and A. Pouquet, Phys. Rev. E 52, 636 (1995).

${ }^{4}$ W. Muller and D. Biskamp, Phys. Rev. Lett. 84, 475 (2000).

${ }^{5}$ R. H. Kraichnan, Phys. Fluids 10, 1417 (1967).

${ }^{6}$ G. K. Batchelor, Phys. Fluids 12, 233 (1969).

${ }^{7}$ U. Frisch, A. Pouquet, P.-L. Sulem, and M. Meneguzzi, J. Mec Theor. Appl. 2, 191 (1983).

${ }^{8}$ A. Pouquet, J. Fluid Mech. 88, 1 (1978).

${ }^{9}$ E. R. Priest and T. Forbes, Magnetic Reconnection: MHD Theory and Applications (Cambridge University Press, New York, 2000).

${ }^{10}$ W. H. Matthaeus and S. L. Lamkin, Phys. Fluids 29, 2513 (1986).

${ }^{11}$ P. Dmitruk and D. O. Gomez, Astrophys. J. 527, L63 (1999).

${ }^{12}$ R. Bruno and V. Carbone, Living Rev. Solar Phys. 2, 4 (2005).

${ }^{13}$ J. W. Bieber, W. Wanner, and W. H. Matthaeus, J. Geophys. Res. 101, 2511, DOI: 10.1029/95JA02588 (1996).

${ }^{14}$ P. Goldreich and S. Sridhar, Astrophys. J. 438, 763 (1995).

${ }^{15}$ J. V. Shebalin, W. H. Matthaeus, and D. C. Montgomery, J. Plasma Phys. 29, 525 (1983).

${ }^{16}$ W. C. Müller, D. Biskamp, and R. Grappin, Phys. Rev. E 67, 066302 (2003).

${ }^{17}$ C. Canuto, M. Y. Hussaini, A. Quarteroni, and T. A. Zang, Spectral Methods in Fluid Mechanics (Springer-Verlag, New York, 1988).

${ }^{18}$ D. Fyfe and D. Montgomery, J. Plasma Phys. 16, 181 (1976).

${ }^{19}$ U. Frisch, A. Pouquet, J. Léorat, and A. Mazure, J. Fluid Mech. 68, 769 (1975).

${ }^{20}$ R. H. Kraichnan and D. Montgomery, Rep. Prog. Phys. 43, 547 (1980).

${ }^{21}$ C. Cichowlas, P. Bonaiti, F. Debbasch, and M. Brachet, Phys. Rev. Lett. 95, 264502 (2005).

${ }^{22}$ U. Frisch, S. Kurien, R. Pandit, W. Pauls, S. S. Ray, A. Wirth, and J.-Z. Zhu, Phys. Rev. Lett. 101, 144501 (2008).

${ }^{23}$ S. Servidio, W. H. Matthaeus, and P. Dmitruk, Phys. Rev. Lett. 100, 095005 (2008); W. H. Matthaeus, A. Pouquet, P. D. Mininni, P. Dmitruk, and B. Breech, ibid. 100, 085003 (2008).

${ }^{24}$ G. Dar, M. K. Verma, and V. Eswaran, Physica D 157, 207 (2001).

${ }^{25}$ A. Alexakis, P. D. Mininni, and A. Pouquet, Phys. Rev. E 72, 046301 (2005).

${ }^{26}$ J. A. Domaradzki and D. Carati, Phys. Fluids 19, 085112 (2007).

${ }^{27}$ O. Debliquy, M. K. Verma, and D. Carati, Phys. Plasmas 12, 042309 (2005).

${ }^{28}$ L. J. Milano, W. H. Matthaeus, B. Breech, and C. W. Smith, Phys. Rev. E 65, 026310 (2002).

${ }^{29}$ R. Grauer and C. Marliani, Phys. Rev. Lett. 84, 4850 (2000).

${ }^{30}$ S. Servidio, W. H. Matthaeus, and V. Carbone, Phys. Plasmas 15, 042314 (2008).

${ }^{31}$ J. Merrifield, S. C. Chapman, and R. O. Dendy, Phys. Plasmas 14, 012301 (2007); J. Merrifield, W. C. Muller, S. C. Chapman, and R. O. Dendy, ibid. 12, 022301 (2005).

${ }^{32}$ E. Lee, M. E. Brachet, A. Pouquet, P. D. Mininni, and D. Rosenberg, Phys. Rev. E 78, 066401 (2008). 